\title{
Efficient Rational Secret Sharing in Standard Communication Networks
}

\author{
Georg Fuchsbauer ${ }^{1, \star}$, Jonathan $\mathrm{Katz}^{2, \star \star}$, and David Naccache ${ }^{1}$ \\ 1 École Normale Supérieure, LIENS - CNRS - INRIA, Paris, France \\ \{georg.fuchsbauer, david. naccache\}@ens.fr \\ 2 University of Maryland, USA \\ jkatz@cs.umd.edu
}

\begin{abstract}
We propose a new methodology for rational secret sharing leading to various instantiations (in both the two-party and multi-party settings) that are simple and efficient in terms of computation, share size, and round complexity. Our protocols do not require physical assumptions or simultaneous channels, and can even be run over asynchronous, pointto-point networks.

We also propose new equilibrium notions (namely, computational versions of strict Nash equilibrium and stability with respect to trembles) and prove that our protocols satisfy them. These notions guarantee, roughly speaking, that at each point in the protocol there is a unique legal message a party can send. This, in turn, ensures that protocol messages cannot be used as subliminal channels, something achieved in prior work only by making strong assumptions on the communication network.
\end{abstract}

\section{Introduction}

The classical problem of $t$-out-of-n secret sharing [28, 5] involves a dealer $D$ who distributes shares of a secret $s$ to players $P_{1}, \ldots, P_{n}$ so that (1) any $t$ or more players can reconstruct the secret without further involvement of the dealer, yet (2) any group of fewer than $t$ players gets no information about $s$. For example, in Shamir's scheme [28] the secret $s$ lies in a finite field $\mathbb{F}$, with $|\mathbb{F}|>n$. The dealer chooses a random polynomial $f(x)$ of degree at most $t-1$ with $f(0)=s$, and gives each player $P_{i}$ the "share" $f(i)$. To reconstruct, $t$ players broadcast their shares and interpolate the polynomial. Any set of fewer than $t$ players has no information about $s$ given their shares.

The implicit assumption in the original formulation of the problem is that each party is either honest or corrupt, and honest parties are all willing to cooperate

The original version of this chapter was revised: The copyright line was incorrect. This has been corrected. The Erratum to this chapter is available at DOI: 10.1007/978-3-642-11799-2_36

* Work supported by EADS, the French ANR-07-SESU-008-01 PAMPA Project, and the European Commission through the ICT Program under Contract ICT-2007216646 ECRYPT II.

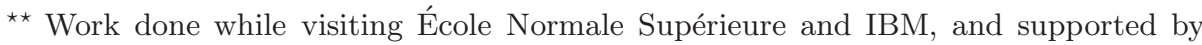
NSF CyberTrust grant \#0830464, NSF CAREER award \#0447075, and DARPA. The contents of this paper do not necessarily reflect the position or the policy of the US Government, and no official endorsement should be inferred.

D. Micciancio (Ed.): TCC 2010, LNCS 5978, pp. 419-436, 2010.

(C) Springer-Verlag Berlin Heidelberg 2010 
when reconstruction of the secret is desired. Beginning with the work of Halpern and Teague [13], protocols for secret sharing and other cryptographic tasks have begun to be re-evaluated in a game-theoretic light (see [7, 16] for an overview of work in this direction). In this setting, parties are neither honest nor corrupt but are instead viewed as rational and are assumed (only) to act in their own self-interest.

Under natural assumptions regarding the utilities of the parties, standard secret-sharing schemes completely fail. For example, assume as in [13] that all players want to learn the secret above all else, but otherwise prefer that no other players learn the secret. (Later, we will treat the utilities of the players more precisely.) For $t$ parties to reconstruct the secret in Shamir's scheme, each party is supposed to broadcast their share simultaneously. It is easy to see, however, that each player does no worse (and potentially does better) by withholding their share no matter what the other players do. Consider $P_{1}$ : If fewer than $t-1$ players reveal their shares, $P_{1}$ does not learn the secret regardless of whether $P_{1}$ reveals his share or not. If more than $t-1$ other players reveal their shares, then everyone learns the secret and $P_{1}$ 's actions again have no effect. On the other hand, if exactly $t-1$ other players reveal their shares, then $P_{1}$ learns the secret (using his share) but prevents other players from learning the secret by not revealing his own share. The result is that if all players are rational then no one will broadcast their share and the secret will not be reconstructed.

Several works 13, 11, 24, 1, 18, 19, 27, 26, 4 have focused on designing rational secret-sharing protocols immune to the above problem. Protocols for rational secret sharing also follow from the more general results of Lepinski et al. 20,21, 15. 14. Each of these works has some or all of the following disadvantages:

On-line dealer or trusted/honest parties. Halpern and Teague [13] introduced a general approach to solving the problem that has been followed in most subsequent work. Their solution, however, requires the continual involvement of the dealer, even after the initial shares have been distributed. (The HalpernTeague solution also applies only when $t, n \geq 3$.) Ong et al. [27 assume that sufficiently many parties behave honestly during the reconstruction phase.

Computational inefficiency. To eliminate the on-line dealer, several schemes rely on multiple invocations of protocols for generic secure multi-party computation 11 24:18.4. It is unclear whether computationally efficient protocols with suitable functionality can be designed. The solutions of [20, 21, 15, 14, though following a different high-level approach, also rely on generic secure multi-party computation.

Strong communication models. All prior schemes for $n>2$ assume broadcast. The solutions in 13, 11,24,1] assume simultaneous broadcast which means that parties must decide on what value (if any) to broadcast in a given round before observing the values broadcast by other parties. The solutions of [20, 21 15, 14 rely on physical assumptions such as secure envelopes and ballot boxes.

Kol and Naor 18 show how to avoid simultaneous broadcast, at the cost of increasing the round complexity by a (multiplicative) factor linear in the size of 
the domain from which the secret is chosen; this approach cannot (efficiently) handle secrets of super-logarithmic length. Subsequent work by Kol and Naor [19] (see also 4) shows how to avoid the assumption of simultaneous broadcast at the expense of increasing the round complexity by a (multiplicative) factor of $t$. We provide a detailed comparison of our results to those of [19] in Section [1.3]

\section{$1.1 \quad$ Our Results}

We show protocols for both 2-out-of-2 and $t$-out-of- $n$ secret sharing (resilient to coalitions of size $t-1$ ) that do not suffer from any of the drawbacks mentioned above. We do not assume an on-line dealer or any trusted/honest parties, nor do we resort to generic secure multi-party computation. Our protocols are (arguably) simpler than previous solutions; they are also extremely efficient in terms of round complexity, share size, and required computation.

The primary advantage of our protocols, however, is that they do not require broadcast or simultaneous communication but can instead rely on synchronous (but non-simultaneous) point-to-point channels. Recall that all prior schemes for $n>2$ assume broadcast; furthermore, the obvious approach of simulating broadcast by running a broadcast protocol over a point-to-point network will not, in general, work in the rational setting. Going further, we show that our protocol can be adapted for asynchronous point-to-point networks (with respect to a natural extension of the model for rational secret sharing), thus answering a question that had been open since the work of Halpern and Teague [13.

We also introduce two new equilibrium notions and prove that our protocols satisfy them. (A discussion of game-theoretic equilibrium notions used in this and prior work is given in Section 2.2.) The first notion we introduce is a computational version of strict Nash equilibrium. A similar notion was put forth by Kol and Naor [19], but they used an information-theoretic version of strict Nash and showed some inherent limitations of doing so. As in all of cryptography, we believe computational relaxations are meaningful and should be considered; doing so allows us to circumvent the limitations that hold in the informationtheoretic case. We also formalize a notion of stability with respect to trembles, motivated by [16; a different formalization of this notion, with somewhat different motivation, is given in [27].

Our definitions effectively rule out "signalling" via subliminal channels in the protocol. In fact, our protocols ensure that, at every point, there is a unique legal message each party can send. This prevents a party from outwardly appearing to follow the protocol while subliminally communicating (or trying to organize collusion) with other parties. Preventing subliminal communication is an explicit goal of some prior work (e.g., [15,21,3, 2]), which achieved it only by relying on physical assumptions [15,21] or non-standard network models [3, 2].

\subsection{Overview of Our Approach}

We follow the same high-level approach as in 13, 11, 24, 1, 18, 19, 4. Our reconstruction protocol proceeds in a sequence of "fake" iterations followed by a single "real" iteration. Roughly speaking: 
- In the real iteration, everyone learns the secret (assuming everyone follows the protocol).

- In a fake iteration, no information about the secret is revealed.

- No party can tell, in advance, whether the next iteration will be real or fake.

The iteration number $i^{*}$ of the real iteration is chosen according to a geometric distribution with parameter $\beta \in(0,1)$ (where $\beta$ depends on the players' utilities). To reconstruct the secret, parties run a sequence of iterations until the real iteration is identified, at which point all parties output the secret. If some party fails to follow the protocol, all parties abort. Intuitively, it is rational for $P_{i}$ to follow the protocol as long as the expected gain of deviating, which is positive only if $P_{i}$ aborts exactly in iteration $i^{*}$, is outweighed by the expected loss if $P_{i}$ aborts before iteration $i^{*}$.

In most prior work 1124 18], a secure multi-party computation is performed in each iteration to determine whether the given iteration should be real or fake. Instead we use the following approach, described in the 2-out-of-2 case (we omit some technical details in order to focus on the main idea): The dealer $D$ chooses $i^{*}$ from the appropriate distribution in advance, at the time of sharing. The dealer then generates two key-pairs $\left(v k_{1}, s k_{1}\right),\left(v k_{2}, s k_{2}\right)$ for a verifiable random function (VRF) [25], where $v k$ represents a verification key and $s k$ represents a secret key, and we denote by $\operatorname{VRF}_{s k}(x)$ the evaluation of the VRF on input $x$ using secret key sk. (See Appendix A for definitions of VRFs.) The dealer gives the verification keys to both parties, gives $s k_{1}$ to $P_{1}$, and gives $s k_{2}$ to $P_{2}$. It also gives $s_{1}=s \oplus \operatorname{VRF}_{s k_{2}}\left(i^{*}\right)$ to $P_{1}$, and $s_{2}=s \oplus \operatorname{VRF}_{s k_{1}}\left(i^{*}\right)$ to $P_{2}$. Each iteration consists of one message from each party: in iteration $i$, party $P_{1}$ sends $\operatorname{VRF}_{s k_{1}}(i)$ while $P_{2}$ sends $\operatorname{VRF}_{s k_{2}}(i)$. Observe that a fake iteration reveals nothing about the secret, in a computational sense. Furthermore, neither party can identify the real iteration in advance. (The description above relies on VRFs. We show that, in fact, trapdoor permutations suffice.)

To complete the protocol, we need to provide a way for parties to identify the real iteration. Previous work 11,24, 1, 18 allows parties to identify the real iteration as soon as it occurs. We could use this approach for our protocol as well if we assumed simultaneous channels, since then each party must decide on its current-iteration message before it learns whether the current iteration is real or fake. When simultaneous channels are not available, however, this approach is vulnerable to an obvious rushing strategy.

Motivated by recent work on fairness (in the malicious setting) 10, 12, we suggest the following, new approach: delay the signal indicating whether a given iteration is real or fake until the following iteration. As before, a party cannot risk aborting until it is sure that the real iteration has occurred; the difference is that now, once a party learns that the real iteration occurred, the real iteration is over and all parties can reconstruct the secret. This eliminates the need for simultaneous channels, while adding only a single round. This approach can be adapted for $t$-out-of- $n$ secret sharing and can be shown to work even when parties communicate over asynchronous, point-to-point channels. 
Our protocol assumes parties have no auxiliary information about the secret $s$. (If simultaneous channels are assumed, then our protocol does tolerate auxiliary information about s.) We believe there are settings where this assumption is valid, and that understanding this case sheds light on the general question of rational computation. Prior work in the non-simultaneous model [18, 19] also fails in the presence of auxiliary information, and in fact this is inherent [4].

\subsection{Comparison to the Kol-Naor Scheme}

The only prior rational secret-sharing scheme that assumes no honest parties, is computationally efficient, and does not require simultaneous broadcast or physical assumptions is that of Kol and Naor [19] (an extension of this protocol is given in [4]). They also use the strict Nash solution concept and so their work provides an especially good point of comparison. Our protocols have the following advantages with respect to theirs:

Share size. In the Kol-Naor scheme, the shares of the parties have unbounded length. While not a significant problem in its own right, this is problematic when rational secret sharing is used as a sub-routine for rational computation of general functions. (See 18.) Moreover, the expected length of the parties' shares in their 2-out-of-2 scheme is $O\left(\beta^{-1} \cdot(|s|+k)\right.$ ) (where $k$ is a security parameter), whereas shares in our scheme have size $|s|+O(k)$.

Round complexity. The version of the Kol-Naor scheme that does not rely on simultaneous broadcast [19, Section 6] has expected round complexity $O\left(\beta^{-1} \cdot t\right)$, whereas our protocol has expected round complexity $O\left(\beta^{-1}\right)$. (The value of $\beta$ is roughly the same in both cases.)

Resistance to coalitions. For the case of $t$-out-of- $n$ secret sharing, the KolNaor scheme is susceptible to coalitions of two or more players. We show $t$-outof- $n$ secret-sharing protocols resilient to coalitions of up to $(t-1)$ parties; see Section $\square$ for further details.

Avoiding broadcast. The Kol-Naor scheme for $n>2$ assumes synchronous broadcast, whereas our protocols work even if parties communicate over an asynchronous, point-to-point network.

\section{Model and Definitions}

We denote the security parameter by $k$. A function $\epsilon: \mathbb{N} \rightarrow \mathbb{R}$ is negligible if for all $c>0$ there is a $k_{c}>0$ such that $\epsilon(k)<1 / k^{c}$ for all $k>k_{c}$; let negl denote a generic negligible function. We say $\epsilon$ is noticeable if there exist $c, k_{c}$ such that $\epsilon(k)>1 / k^{c}$ for all $k>k_{c}$.

We define our model and then describe the game-theoretic concepts used. Even readers familiar with prior work in this area should skim the next few sections, since we formalize certain aspects of the problem slightly differently from prior work, and define new equilibrium notions. 


\subsection{Secret Sharing and Players' Utilities}

A $t$-out-of-n secret-sharing scheme for domain $\mathcal{S}$ (with $|\mathcal{S}|>1$ ) is a two-phase protocol carried out by a dealer $D$ and a set of $n$ parties $P_{1}, \ldots, P_{n}$. In the first phase (the sharing phase), the dealer chooses a secret $s \in \mathcal{S}$. Based on this secret and a security parameter $1^{k}$, the dealer generates shares $s_{1}, \ldots, s_{n}$ and gives $s_{i}$ to player $P_{i}$. In the second phase (the reconstruction phase), some set $I$ of $t^{*} \geq t$ active parties jointly reconstruct $s$. We impose the following requirements:

Secrecy. The shares of any $t-1$ parties reveal nothing about $s$, in an informationtheoretic sense.

Correctness. For any set $I$ of $t^{*} \geq t$ parties who run the reconstruction phase honestly, the correct secret $s$ will be reconstructed, except possibly with probability negligible in $k$.

The above views parties as either malicious or honest. To model rationality, we consider players' utilities. Given a set $I$ of $t^{*} \geq t$ parties active during the reconstruction phase, let the outcome $o$ of the reconstruction phase be a vector of length $t^{*}$ with $o_{i}=1$ iff the output of $P_{i}$ is equal to the initial secret $s$ (i.e., $P_{i}$ "learned the secret"). We consider a party to have learned the secret $s$ if and only if it outputs $s$, and do not care whether that party "really knows" the secret or not. In particular, a party who outputs a random value in $\mathcal{S}$ without running the reconstruction phase at all "learns" the secret with probability $1 /|\mathcal{S}|$. We model the problem this way for two reasons:

1. Our formulation lets us model a player learning partial information about the secret, something not reflected in prior work. In particular, partial information that increases the probability with which a party outputs the correct secret increases that party's expected utility.

2. It is difficult, in general, to formally model what it means for a party to "really" learn the secret, especially when considering arbitrary protocols and behaviors. Our notion is also better suited for a computational setting, where a party might "know" the secret from an information-theoretic point of view yet be unable to output it.

Let $\mu_{i}(o)$ be the utility of player $P_{i}$ for the outcome $o$. Following [13] and most subsequent work (an exception is 4]), we make the following assumptions about the utility functions of the players:

- If $o_{i}>o_{i}^{\prime}$, then $\mu_{i}(o)>\mu_{i}\left(o^{\prime}\right)$.

- If $o_{i}=o_{i}^{\prime}$ and $\sum_{i} o_{i}<\sum_{i} o_{i}^{\prime}$, then $\mu_{i}(o)>\mu_{i}\left(o^{\prime}\right)$.

That is, player $P_{i}$ first prefers outcomes in which he learns the secret; otherwise, $P_{i}$ prefers strategies in which the fewest number of other players learn the secret. For simplicity, in our analysis we distinguish three cases for the outcome $o$, described from the point of view of $P_{i}$ (we could also work with utilities satisfying the more general constraints above, as long as utilities are known): 
1. If $P_{i}$ learns the secret and no other player does, then $\mu_{i}(o) \stackrel{\text { def }}{=} U^{+}$.

2. If $P_{i}$ learns the secret and at least one other player does also, then $\mu_{i}(o) \stackrel{\text { def }}{=} U$.

3. If $P_{i}$ does not learn the secret, then $\mu_{i}(o) \stackrel{\text { def }}{=} U^{-}$.

Our conditions impose $U^{+}>U>U^{-}$. Define

$$
U_{\text {random }} \stackrel{\text { def }}{=} \frac{1}{|\mathcal{S}|} \cdot U^{+}+\left(1-\frac{1}{|\mathcal{S}|}\right) \cdot U^{-} ;
$$

this is the expected utility of a party who outputs a random guess for the secret (assuming other parties abort without any output, or with the wrong output). We will also assume that $U>U_{\text {random; }}$ otherwise, players have (almost) no incentive to run the reconstruction phase at all.

In contrast to 4, we make no distinction between outputting the wrong secret and outputting a special "don't know" symbol; both are considered a failure to output the correct secret. By adapting techniques from their work, however, we can incorporate this distinction as well (as long as the relevant utilities are known). See Remark 2 in Section 3

Strategies in our context refer to probabilistic polynomial-time interactive Turing machines. Given a vector of strategies $\boldsymbol{\sigma}$ for $t^{*}$ parties active in the reconstruction phase, let $u_{i}(\boldsymbol{\sigma})$ denote the expected utility of $P_{i}$. (The expected utility is a function of the security parameter $k$.) This expectation is taken over the initial choice of $s$ (which we will always assume to be uniform), the dealer's randomness, and the randomness of the players' strategies. Following the standard game-theoretic notation, we define $\boldsymbol{\sigma}_{-i} \stackrel{\text { def }}{=}\left(\sigma_{1}, \ldots, \sigma_{i-1}, \sigma_{i+1}, \ldots, \sigma_{t^{*}}\right)$ and $\left(\sigma_{i}^{\prime}, \boldsymbol{\sigma}_{-i}\right) \stackrel{\text { def }}{=}\left(\sigma_{1}, \ldots, \sigma_{i-1}, \sigma_{i}^{\prime}, \sigma_{i+1}, \ldots, \sigma_{t^{*}}\right)$; that is, $\left(\sigma_{i}^{\prime}, \boldsymbol{\sigma}_{-i}\right)$ denotes the strategy vector $\boldsymbol{\sigma}$ with $P_{i}^{\prime}$ 's strategy changed to $\sigma_{i}^{\prime}$.

\subsection{Notions of Game-Theoretic Equilibria: A Discussion}

The starting point for any discussion of game-theoretic equilibria is the Nash equilibrium. Roughly speaking, a protocol induces a Nash equilibrium if no party gains any advantage by deviating from the protocol, as long as all other parties follow the protocol. (In a computational Nash equilibrium, no efficient deviation confers any advantage.) As observed by Halpern and Teague [13], however, the Nash equilibrium concept is too weak for rational secret sharing. Halpern and Teague suggest, instead, to design protocols that induce a Nash equilibrium surviving iterated deletion of weakly dominated strategies; this notion was used in subsequent work of 11,24, 11.

The notion of surviving iterated deletion, though, is also problematic in several respects. Kol and Naor [19] show a secret-sharing protocol that is "intuitively bad" yet technically satisfies the definition because no strategy weakly dominates any other. Also, a notion of surviving iterated deletion taking computational issues into account has not yet been defined (and doing so appears difficult). See 16,17 for other arguments against this notion. 
Motivated by these drawbacks (and more), researchers have proposed other strengthenings of the Nash equilibrium concept [16, 18, 19]. Kol and Naor define resistance to backward induction 18, everlasting equilibrium, and strict Nash equilibrium [19. The latter two notions are defined information-theoretically, and are overly conservative in that they rule out some "natural" protocols using cryptography. Nevertheless, the notion of strict Nash equilibrium is appealing. A protocol is in Nash equilibrium if no deviations are advantageous; it is in strict Nash equilibrium if all deviations are disadvantageous. Put differently, in the case of a Nash equilibrium there is no incentive to deviate whereas for a strict Nash equilibrium there is an incentive not to deviate.

Another advantage of strict Nash is that protocols satisfying this notion deter subliminal communication: since any (detectable) deviation from the protocol results in lower utility (when other parties follow the protocol), a party who tries to use protocol messages as a covert channel risks losing utility if there is any reasonable probability that other players are following the protocol.

We propose here a computational version of strict Nash equilibrium. Our definition retains the intuitive appeal of strict Nash, while meaningfully taking computational limitations into account. Moreover, our protocols satisfy the following, stronger condition: at every point in the protocol, there is a unique legal message that each party can send. Our protocols thus rule out subliminal communication in a strong sense, an explicit goal in prior work [20, 22, 21, 3].

We also define a computational notion of stability with respect to trembles. Intuitively, stability with respect to trembles models players' uncertainty about other parties' behavior, and guarantees that even if a party $P_{i}$ believes that other parties might play some arbitrary strategy with small probability $\delta$ (but follow the protocol with probability $1-\delta$ ), there is still no better strategy for $P_{i}$ than to follow the protocol. Our formulation of this notion follows the general suggestion of Katz 16, but we flesh out the (non-trivial) technical details. Another formulation (trembling-hand perfect equilibrium), with somewhat different motivation, is discussed in [27.

As should be clear, determining the "right" game-theoretic notions for rational secret sharing is the subject of ongoing research. We do not suggest that the definitions proposed here are the only ones to consider, but we do believe they contribute to our understanding of the problem.

\subsection{Definitions of Game-Theoretic Equilibria}

We focus on the two-party case; the multi-party case is treated in the full version of this work 9 . Here, $\Pi$ is a 2-out-of-2 secret-sharing scheme and $\sigma_{i}$ is the prescribed action of $P_{i}$ in the reconstruction phase.

Definition 1. $\Pi$ induces a computational Nash equilibrium if for any PPT strategy $\sigma_{1}^{\prime}$ of $P_{1}$ we have $u_{1}\left(\sigma_{1}^{\prime}, \sigma_{2}\right) \leq u_{1}\left(\sigma_{1}, \sigma_{2}\right)+\operatorname{negl}(k)$, and similarly for $P_{2}$.

Our definitions of strict Nash and resistance to trembles require us to first define what it means to "follow a protocol". This is non-trivial since a different Turing machine $\rho_{1}$ might be "functionally identical" to the prescribed strategy $\sigma_{1}$ as 
far as the protocol is concerned: for example, $\rho_{1}$ may be the same as $\sigma_{1}$ except that it first performs some useless computation; the strategies may be identical except that $\rho_{1}$ uses pseudorandom coins instead of random coins; or, the two strategies may differ in the message(s) they send after the protocol ends. In any of these cases we would like to say that $\rho_{1}$ is essentially "the same" as $\sigma_{1}$. This motivates the following definition, stated for the case of a deviating $P_{1}$ (with an analogous definition for a deviating $P_{2}$ ):

Definition 2. Define the random variable view ${ }_{2}^{\Pi}$ as follows:

$P_{1}$ and $P_{2}$ interact, following $\sigma_{1}$ and $\sigma_{2}$, respectively. Let trans denote the messages sent by $P_{1}$ but not including any messages sent by $P_{1}$ after it writes to its (write-once) output tape. Then view ${ }_{2}^{\Pi}$ includes the information given by the dealer to $P_{2}$, the random coins of $P_{2}$, and the (partial) transcript trans.

Fix a strategy $\rho_{1}$ and an algorithm $T$. Define the random variable view ${ }_{2}^{T, \rho_{1}}$ as follows:

$P_{1}$ and $P_{2}$ interact, following $\rho_{1}$ and $\sigma_{2}$, respectively. Let trans denote the messages sent by $P_{1}$. Algorithm $T$, given the entire view of $P_{1}$, outputs an arbitrary truncation trans' of trans. (That is, it defines a cut-off point and deletes any messages sent after that point.) Then view $_{2}^{T, \rho_{1}}$ includes the information given by the dealer to $P_{2}$, the random coins of $P_{2}$, and the (partial) transcript trans'.

Strategy $\rho_{1}$ yields equivalent play with respect to $\Pi$, denoted $\rho_{1} \approx \Pi$, if there exists a PPT algorithm $T$ such that for all PPT distinguishers $D$

$$
\left|\operatorname{Pr}\left[D\left(1^{k}, \operatorname{view}_{2}^{T, \rho_{1}}\right)=1\right]-\operatorname{Pr}\left[D\left(1^{k}, \operatorname{view}_{2}^{\Pi}\right)=1\right]\right| \leq \operatorname{neg}(k) .
$$

We write $\rho_{1} \not \approx \Pi$ if $\rho_{1}$ does not yield equivalent play with respect to $\Pi$. Note that $\rho_{1}$ can yield equivalent play with respect to $\Pi$ even if (1) it differs from the prescribed strategy when interacting with some other strategy $\sigma_{2}^{\prime}$ (we only care about the behavior of $\rho_{1}$ when the other party runs $\Pi$ ); (2) it differs from the prescribed strategy in its local computation or output; and (3) it differs from the prescribed strategy after $P_{1}$ computes its output. This last point models the fact that we cannot force $P_{1}$ to send "correct" messages once, as far as $P_{1}$ is concerned, the protocol is finished.

We now define the notion that detectable deviations from the protocol decrease a player's utility.

\section{Definition 3. $\Pi$ induces a computational strict Nash equilibrium if}

1. $\Pi$ induces a computational Nash equilibrium;

2. For any PPT strategy $\sigma_{1}^{\prime}$ with $\sigma_{1}^{\prime} \not \approx \Pi$, there is a $c>0$ such that $u_{1}\left(\sigma_{1}, \sigma_{2}\right) \geq$ $u_{1}\left(\sigma_{1}^{\prime}, \sigma_{2}\right)+1 / k^{c}$ for infinitely many values of $k$ (with an analogous requirement for a deviating $\left.P_{2}\right)$.

We next turn to defining stability with respect to trembles. We say that $\rho_{i}$ is $\delta$-close to $\sigma_{i}$ if $\rho_{i}$ takes the following form: with probability $1-\delta$ party $P_{i}$ plays 
$\sigma_{i}$, while with probability $\delta$ it follows an arbitrary PPT strategy $\sigma_{i}^{\prime}$. (In this case, we refer to $\sigma_{i}^{\prime}$ as the residual strategy of $\rho_{i}$.) The notion of $\delta$-closeness is meant to model a situation in which $P_{i}$ plays $\sigma_{i}$ "most of the time," but with some (small) probability plays some other arbitrary strategy.

Intuitively, a pair of strategies $\left(\sigma_{1}, \sigma_{2}\right)$ is stable with respect to trembles if $\sigma_{1}$ (resp., $\sigma_{2}$ ) remains a best response even if the other party plays a strategy other than $\sigma_{2}$ (resp., $\sigma_{1}$ ) with some small (but noticeable) probability $\delta$. As in the case of strict Nash equilibrium, this notion is difficult to define formally because of the possibility that one party can do better (in case the other deviates) by performing some (undetectable) local computation 1 Our definition essentially requires that this is the only way for either party to do better and so, in particular, each party will (at least outwardly) continue to follow the protocol until the other deviates. The fact that the prescribed strategies are in Nash equilibrium ensures that any (polynomial-time) local computation performed by either party is of no benefit as long as the other party follows the protocol.

Definition 4. $\Pi$ induces a computational Nash equilibrium that is stable with respect to trembles if

1. $\Pi$ induces a computational Nash equilibrium;

2. There is a noticeable function $\delta$ such that for any PPT strategy $\rho_{2}$ that is $\delta$-close to $\sigma_{2}$, and any PPT strategy $\rho_{1}$, there exists a PPT strategy $\sigma_{1}^{\prime} \approx \Pi$ such that $u_{1}\left(\rho_{1}, \rho_{2}\right) \leq u_{1}\left(\sigma_{1}^{\prime}, \rho_{2}\right)+\operatorname{negl}(k)$ (with an analogous requirement for the case of deviations by $\left.P_{2}\right)$.

Stated differently, even if a party $P_{i}$ believes that the other party might play a different strategy with some small probability $\delta$, there is still no better strategy for $P_{i}$ than to outwardly follow the protoco (while possibly performing some additional local computation).

\section{Rational Secret Sharing: The 2-Out-of-2 Case}

Let $\mathcal{S}=\{0,1\}^{\ell}$ be the domain of the secret. Let (Gen, Eval, Prove, Vrfy) be a VRF with range $\{0,1\}^{\ell}$, and let $\left(\mathrm{Gen}^{\prime}, \mathrm{Eval}^{\prime}\right.$, Prove $\left.^{\prime}, \mathrm{Vrfy}^{\prime}\right)$ be a VRF with range $\{0,1\}^{k}$. Protocol $\Pi$ is defined as follows:

Sharing phase. Let $s$ denote the secret. The dealer chooses an integer $i^{*} \in \mathbb{N}$ according to a geometric distribution with parameter $\beta$, where $\beta$ is a constant that depends on the players' utilities but is independent of the security parameter; we discuss how to set $\beta$ below. We assume $i^{*}<2^{k}-1$ since this occurs with all but negligible probability. (Technically, if $i^{*} \geq 2^{k}-1$ the dealer can just send a special error message to each party.)

The dealer first computes the keys $\left(p k_{1}, s k_{1}\right),\left(p k_{2}, s k_{2}\right) \leftarrow \operatorname{Gen}\left(1^{k}\right)$ as well as $\left(p k_{1}^{\prime}, s k_{1}^{\prime}\right),\left(p k_{2}^{\prime}, s k_{2}^{\prime}\right) \leftarrow \operatorname{Gen}^{\prime}\left(1^{k}\right)$. Then the dealer computes:

\footnotetext{
${ }^{1}$ As a trivial example, consider the case where with probability $\delta$ one party just sends its share to the other.

2 Specifically, for any strategy $\rho_{i}$ that does not yield equivalent play w.r.t. $\Pi$, there is a strategy $\sigma_{i}^{\prime}$ that does yield equivalent play w.r.t. $\Pi$ and performs about as well.
} 
- share $_{1}:=\operatorname{Eval}_{s k_{2}}\left(i^{*}\right) \oplus s$ and share $2:=\operatorname{Eval}_{s k_{1}}\left(i^{*}\right) \oplus s$;

- signal $_{1}:=\mathrm{Eval}_{s k_{2}^{\prime}}^{\prime}\left(i^{*}+1\right)$ and signal $2:=\mathrm{Eval}_{s k_{1}^{\prime}}^{\prime}\left(i^{*}+1\right)$.

Finally, the dealer gives to $P_{1}$ the values $\left(s k_{1}, s k_{1}^{\prime}, p k_{2}, p k_{2}^{\prime}\right.$, share ${ }_{1}$, signal $\left.{ }_{1}\right)$, and gives to $P_{2}$ the values $\left(s k_{2}, s k_{2}^{\prime}, p k_{1}, p k_{1}^{\prime}\right.$, share 2 , signal $\left.{ }_{2}\right)$.

As written, the share given to each party only hides $s$ in a computational sense. Nevertheless, information-theoretic secrecy is easy to achieve; see Remark 1 at the end of this section.

\section{Reconstruction phase}

At the outset, $P_{1}$ chooses $s_{1}^{(0)}$ uniformly from $\mathcal{S}=\{0,1\}^{\ell}$ and $P_{2}$ chooses $s_{2}^{(0)}$ the same way. Then in each iteration $i=1, \ldots$, the parties do the following:

( $P_{2}$ sends message to $\left.P_{1}:\right) P_{2}$ computes

$-y_{2}^{(i)}:=\operatorname{Eval}_{s k_{2}}(i), \pi_{2}^{(i)}:=\operatorname{Prove}_{s k_{2}}(i)$

$-z_{2}^{(i)}:=\operatorname{Eval}_{s k_{2}^{\prime}}^{\prime}(i), \bar{\pi}_{2}^{(i)}:=\operatorname{Prove}_{s k_{2}^{\prime}}^{\prime}(i)$.

It then sends $\left(y_{2}^{(i)}, \pi_{2}^{(i)}, z_{2}^{(i)}, \bar{\pi}_{2}^{(i)}\right)$ to $P_{1}$.

$\left(P_{1}\right.$ receives message from $\left.P_{2}:\right) P_{1}$ receives $\left(y_{2}^{(i)}, \pi_{2}^{(i)}, z_{2}^{(i)}, \bar{\pi}_{2}^{(i)}\right)$ from $P_{2}$. If $P_{2}$ does not send anything, or if $\operatorname{Vrfy}_{p k_{2}}\left(i, y_{2}^{(i)}, \pi_{2}^{(i)}\right)=0$ or $\operatorname{Vrfy}_{p k_{2}^{\prime}}^{\prime}\left(i, z_{2}^{(i)}, \bar{\pi}_{2}^{(i)}\right)=0$, then $P_{1}$ outputs $s_{1}^{(i-1)}$ and halts.

If signal ${ }_{1} \stackrel{?}{=} z_{2}^{(i)}$ then $P_{1}$ outputs $s_{1}^{(i-1)}$, sends its iteration- $i$ message to $P_{2}$ (see below), and halts. Otherwise, it sets $s_{1}^{(i)}:=$ share $_{1} \oplus y_{2}^{(i)}$ and continues.

( $P_{1}$ sends message to $\left.P_{2}:\right) P_{1}$ computes

$-y_{1}^{(i)}:=\operatorname{Eval}_{s k_{1}}(i), \pi_{1}^{(i)}:=\operatorname{Prove}_{s k_{1}}(i)$

$-z_{1}^{(i)}:=\operatorname{Eval}_{s k_{1}^{\prime}}^{\prime}(i), \bar{\pi}_{1}^{(i)}:=\operatorname{Prove}_{s k_{1}^{\prime}}^{\prime}(i)$.

It then sends $\left(y_{1}^{(i)}, \pi_{1}^{(i)}, z_{1}^{(i)}, \bar{\pi}_{1}^{(i)}\right)$ to $P_{2}$.

$\left(P_{2}\right.$ receives message from $\left.P_{1}:\right) P_{2}$ receives $\left(y_{1}^{(i)}, \pi_{1}^{(i)}, z_{1}^{(i)}, \bar{\pi}_{1}^{(i)}\right)$ from $P_{1}$. If $P_{1}$ does not send anything, or if $\operatorname{Vrfy}_{p k_{1}}\left(i, y_{1}^{(i)}, \pi_{1}^{(i)}\right)=0$ or $\operatorname{Vrfy}_{p k_{1}^{\prime}}^{\prime}\left(i, z_{1}^{(i)}, \bar{\pi}_{1}^{(i)}\right)=0$, then $P_{2}$ outputs $s_{2}^{(i-1)}$ and halts.

If signal ${ }_{2} \stackrel{?}{=} z_{1}^{(i)}$ then $P_{2}$ outputs $s_{2}^{(i-1)}$ and halts. Otherwise, it sets $s_{2}^{(i)}:=$ share $_{2} \oplus y_{1}^{(i)}$ and continues.

Fig. 1. The reconstruction phase of secret-sharing protocol $\Pi$.

Reconstruction phase. A high-level overview of the protocol was given in Section 1.1] and we give the formal specification in Figure 10 The reconstruction phase proceeds in a series of iterations, where each iteration consists of one message sent by each party. Although these messages could be sent at the same time (since they do not depend on each other), we do not want to assume simultaneous communication and therefore simply require $P_{2}$ to communicate first in 
each iteration. (If one were willing to assume simultaneous channels then the protocol could be simplified by having $P_{2}$ send Eval ${ }_{s k_{2}^{\prime}}^{\prime}(i+1)$ at the same time as $\operatorname{Eval}_{s k_{2}}(i)$, and similarly for $P_{1}$.)

We give some intuition as to why the reconstruction phase of $\Pi$ is a computational Nash equilibrium for an appropriate choice of $\beta$. Assume $P_{2}$ follows the protocol, and consider possible deviations by $P_{1}$. (Deviations by $P_{2}$ are even easier to analyze since $P_{2}$ goes first in every iteration.) $P_{1}$ can abort in iteration $i=i^{*}+1$ (i.e., as soon as it receives $z_{2}^{(i)}=$ signal $_{1}$ ), or it can abort in some iteration $i<i^{*}+1$. In the first case $P_{1}$ "knows" that it learned the dealer's secret in the preceding iteration (that is, in iteration $i^{*}$ ) and can thus output the correct secret; however, $P_{2}$ will output $s_{2}^{\left(i^{*}\right)}=s$ and so learns the secret as well. So $P_{1}$ does not increase its utility beyond what it would achieve by following the protocol. In the second case, when $P_{1}$ aborts in some iteration $i<i^{*}+1$, the best strategy $P_{1}$ can adopt is to output $s_{1}^{(i)}$ and hope that $i=i^{*}$. The expected utility that $P_{1}$ obtains by following this strategy can be calculated as follows:

- $P_{1}$ aborts exactly in iteration $i=i^{*}$ with probability $\beta$. Then $P_{1}$ gets utility at most $U^{+}$.

- When $i<i^{*}$, player $P_{1}$ has "no information" about $s$ and so the best it can do is guess. The expected utility of $P_{1}$ in this case is thus at most $U_{\text {random }}$ (cf. Equation (II)).

Putting everything together, the expected utility of $P_{1}$ following this strategy is at most $\beta \times U^{+}+(1-\beta) \times U_{\text {random. Since }} U_{\text {random }}<U$ by assumption, it is possible to set $\beta$ so that the expected utility of this strategy is strictly less than $U$, in which case $P_{1}$ has no incentive to deviate.

That $\Pi$ induces a strict computational Nash equilibrium (which is also stable with respect to trembles) follows since there is always a unique valid message a party can send; anything else is treated as an abort. A proof of the following theorem appears in the full version of this work 9 .

Theorem 1. If $\beta>0$ and $U>\beta \cdot U^{+}+(1-\beta) \cdot U_{\text {random }}$, then $\Pi$ induces $a$ computational strict Nash equilibrium that is stable with respect to trembles.

Remark 1. The sharing phase, as described, guarantees computational secrecy only. A generic transformation from any such protocol (with bounded-size shares) to one that achieves information-theoretic secrecy follows: After $D$ generates shares $s_{1}, s_{2}$ in the computationally secure scheme, it chooses random $r_{1}, r_{2}$ and random keys $k_{1}, k_{2}$, and gives to $P_{i}$ the share $\left(s_{i} \oplus r_{i}, k_{i}, r_{3-i}, \operatorname{MAC}_{k_{3-i}}\left(r_{3-i}\right)\right)$, where MAC denotes an information-theoretically secure MAC. The reconstruction phase begins by having the parties exchange $r_{1}$ and $r_{2}$ along with the associated MAC tags, verifying the tags (and aborting if they are incorrect), and then recovering $s_{1}, s_{2}$. They then run the original protocol. It is easy to see that this maintains all the game-theoretic properties of the original protocol.

Remark 2. In the reconstruction phase, as described, one party can cause the other to output an incorrect secret (by aborting early). If the utilities of doing 
so are known, the protocol can be modified to rule out this behavior (in a gametheoretic sense) using the same techniques as in 4, Section 5.2]. Specifically, the dealer can — for each party — designate certain rounds as "completely fake", so that the party will know to output $\perp$ instead of an incorrect secret in case the other party aborts in that round. Using VRFs, this still can be achieved with bounded-size shares. Details will appear in the full version.

\subsection{Using Trapdoor Permutations Instead of VRFs}

The protocol from the previous section can be adapted easily to use trapdoor permutations instead of VRFs. The key observation is that the VRFs in the previous protocol are used only in a very specific way: they applied sequentially to values $1,2, \ldots$. One can therefore use a trapdoor permutation $f$ with associated hardcore bit $h$ to instantiate the VRF in our scheme in the following way: The public key is a description of $f$ along with a random element $y$ in the domain of $f$; the secret key is the trapdoor enabling inversion of $f$. In iteration $i$, the "evaluation" of the VRF on input $i$ is the $\ell$-bit sequence

$$
h\left(f^{-(i-1) \ell-1}(y)\right), h\left(f^{-(i-1) \ell-2}(y)\right), \ldots, h\left(f^{-(i-1) \ell-\ell}(y)\right),
$$

and the "proof" is $\pi_{i}=f^{-(i-1) \ell-\ell}(y)$. Verification can be done using the original point $y$, and can also be done in time independent of $i$ by using $\pi_{i-1}$ (namely, by checking that $\left.f^{\ell}\left(\pi_{i}\right)=\pi_{i-1}\right)$, assuming $\pi_{i-1}$ has already been verified.

The essential properties we need still hold: verifiability and uniqueness of proofs are easy to see, and pseudorandomness still holds with respect to a modified game where the adversary queries $\mathrm{Eval}_{s k}(1), \ldots, \mathrm{Eval}_{s k}(i)$ and then has to guess whether it is given $\mathrm{Eval}_{s k}(i+1)$ or a random string. We omit further details.

\section{Rational Secret Sharing: The $t$-Out-of- $n$ Case}

In this section we describe extensions of our protocol to the $t$-out-of- $n$ case, where we consider deviations by coalitions of up to $t-1$ parties. Formal definitions of game-theoretic notions in the multi-player setting, both for the case of singleplayer deviations as well as coalitions, are fairly straightforward adaptations of the definitions from Section [2.3] and are given in the full version of this work 9].

In describing our protocols we use VRFs for notational simplicity, but all the protocols given here can be instantiated using trapdoor permutations as described in Section 3.1.

A protocol for "exactly $t$-out-of- $n$ " secret sharing. We begin by describing a protocol $\Pi_{t, n}$ for $t$-out-of- $n$ secret sharing that is resilient to coalitions of up to $t-1$ parties under the assumption that exactly $t$ parties are active during the reconstruction phase. (We also require that the coalition be a subset of the active parties.) For now, we assume communication over a synchronous (but not simultaneous) point-to-point network. 


\section{Sharing Phase}

To share a secret $s \in\{0,1\}^{\ell}$, the dealer does the following:

- Choose $r^{*} \in \mathbb{N}$ according to a geometric distribution with parameter $\beta$.

- Generatd VRF keys $\left(p k_{1}, s k_{1}\right), \ldots,\left(p k_{n}, s k_{n}\right) \leftarrow \operatorname{Gen}\left(1^{k}\right)$ followed by $\left(p k_{1}^{\prime}, s k_{1}^{\prime}\right), \ldots,\left(p k_{n}^{\prime}, s k_{n}^{\prime}\right) \leftarrow \operatorname{Gen}^{\prime}\left(1^{k}\right)$.

- Choose random $(t-1)$-degree polynomials $G \in \mathbb{F}_{2^{\ell}}[x]$ and $H \in \mathbb{F}_{2^{k}}[x]$ such that $G(0)=s$ and $H(0)=0$.

- Send $s k_{i}, s k_{i}^{\prime}$ to player $P_{i}$, and send to all parties the following values:

1. $\left\{\left(p k_{j}, p k_{j}^{\prime}\right)\right\}_{1 \leq j \leq n}$

2. $\left\{g_{j}:=G(j) \oplus \operatorname{Eval}_{s k_{j}}\left(r^{*}\right)\right\}_{1 \leq j \leq n}$

3. $\left\{h_{j}:=H(j) \oplus \mathrm{Eval}_{s k_{j}^{\prime}}^{\prime}\left(r^{*}+1\right)\right\}_{1 \leq j \leq n}$

\section{Reconstruction Phase}

Let $I$ be the indices of the $t$ active players. Each party $P_{i}$ (for $i \in I$ ) chooses $s_{i}^{(0)}$ uniformly from $\{0,1\}^{\ell}$. In each iteration $r=1, \ldots$, the parties do:

- For all $i \in I$ (in ascending order), $P_{i}$ sends the following to all players:

$$
\left(y_{i}^{(r)}:=\operatorname{Eval}_{s k_{i}}(r), z_{i}^{(r)}:=\operatorname{Eval}_{s k_{i}^{\prime}}^{\prime}(r), \operatorname{Prove}_{s k_{i}}(r), \operatorname{Prove}_{s k_{i}^{\prime}}^{\prime}(r)\right) .
$$

- If a party $P_{i}$ receives an incorrect proof (or nothing) from any other party $P_{j}$, then $P_{i}$ terminates and outputs $s_{i}^{(r-1)}$. Otherwise:

- $P_{i}$ sets $h_{j}^{(r)}:=h_{j} \oplus z_{j}^{(r)}$ for all $j \in I$, and interpolates a degree$(t-1)$ polynomial $H^{(r)}$ through the $t$ points $\left\{h_{j}^{(r)}\right\}_{j \in I}$. If $H^{(r)}(0) \stackrel{?}{=} 0$ then $P_{i}$ outputs $s_{i}^{(r-1)}$ immediately, and terminates after sending its current-iteration message.

- Otherwise, $P_{i}$ computes $s_{i}^{(r)}$ as follows: set $g_{j}^{(r)}:=g_{j} \oplus y_{j}^{(r)}$ for all $j \in I$. Interpolate a degree- $(t-1)$ polynomial $G^{(r)}$ through the points $\left\{g_{j}^{(r)}\right\}_{j \in I}$, and set $s_{i}^{(r)}:=G^{(r)}(0)$.

${ }^{a}$ Gen outputs VRF keys with range $\{0,1\}^{\ell}$, and Gen' outputs VRF keys with range $\{0,1\}^{k}$.

Fig. 2. Protocol $\Pi_{t, n}$ for "exactly $t$-out-of- $n$ " secret sharing

As in the 2-out-of-2 case, every party is associated with two keys for a VRF. The dealer chooses an iteration $r^{*}$ according to a geometric distribution, and also chooses two random $(t-1)$-degree polynomials $G, H$ (over some finite field) such that $G(0)=s$ and $H(0)=0$. Each party receives blinded versions of all $n$ points $\{G(j), H(j)\}_{j=1}^{n}$ : each $G(j)$ is blinded by the value of $P_{j}$ 's VRF on the input $r^{*}$, and each $H(j)$ is blinded by the value of $P_{j}$ 's VRF on the input $r^{*}+1$. In each iteration $r$, each party is supposed to send to all other parties the value of their VRFs evaluated on the current iteration number $r$; once this is done, every party can interpolate a polynomial to obtain candidate values for $G(0)$ 
and $H(0)$. When $H(0)=0$ parties know the protocol is over, and output the $G(0)$ value reconstructed in the previous iteration. See Figure for details.

Theorem 2. If $\beta>0$ and $U>\beta \cdot U^{+}+(1-\beta) \cdot U_{\text {random }}$, then $\Pi_{t, n}$ induces $a$ $(t-1)$-resilient computational strict Nash equilibrium that is stable with respect to trembles, as long as exactly $t$ parties are active during the reconstruction phase.

A proof is exactly analogous to the proof of Theorem 1

Handling the general case. The prior solution assumes exactly $t$ parties are active during reconstruction. If $t^{*}>t$ parties are active, the "natural" implementation of the protocol — where the lowest-indexed $t$ parties run $\Pi_{t, n}$ and all other parties remain silent - is not a $(t-1)$-resilient computational Nash equilibrium. To see why, let the active parties be $I=\{1, \ldots t+1\}$ and let $\mathcal{C}=\{3, \ldots, t+1\}$ be a coalition of $t-1$ parties. In each iteration $r$, as soon as $P_{1}$ and $P_{2}$ send their values the parties in $\mathcal{C}$ can compute $t+1$ points $\left\{g_{j}^{(r)}\right\}_{j \in I}$. Because of the way these points are constructed, they are guaranteed to lie on a $(t-1)$-degree polynomial when $r=r^{*}$, but are unlikely to lie on a $(t-1)$-degree polynomial when $r<r^{*}$. This gives the parties in $\mathcal{C}$ a way to determine $r^{*}$ as soon as that iteration is reached, at which point they can abort and output the secret while preventing $P_{1}$ and $P_{2}$ from doing the same.

Fortunately, a simple modification works: simply have the dealer run independent instances $\Pi_{t, n}, \Pi_{t+1, n}, \ldots, \Pi_{n, n}$; in the reconstruction phase, the parties run $\Pi_{t^{*}, n}$ where $t^{*}$ denotes the number of active players. It follows as an easy corollary of Theorem 2 that this induces a $(t-1)$-resilient computational strict Nash equilibrium (that is also stable with respect to trembles) regardless of how many parties are active during the reconstruction phase. (As in previous work, we only consider coalitions that are subsets of the parties who are active during reconstruction. The protocol is no longer a computational Nash equilibrium if this is not the case 3 )

Asynchronous networks. Our protocol $\Pi_{t, n}$ can be adapted to work even when the parties communicate over an asynchronous point-to-point network. (In our model of asynchronous networks, messages can be delayed arbitrarily and delivered out of order, but any message that is sent is eventually delivered.) In this case parties cannot distinguish an abort from a delayed message and so we modify the protocol as follows: each party proceeds to the next iteration as soon as it receives $t-1$ valid messages from the previous iteration, and only halts if it receives an invalid message from someone. More formal treatment of the asynchronous case, including a discussion of definitions in this setting and a proof for the preceding protocol, is given in the full version of this work 9].

As before, we can handle the general case by having the dealer run the "exactly $t^{*}$-out-of- $n$ " protocol just described for all values of $t^{*} \in\{t, \ldots, n\}$.

3 This case can be addressed, however, by having the dealer run independent instances of $\Pi_{t, n}$ for all $\left(\begin{array}{l}n \\ t\end{array}\right)$ subsets of size $t$; to reconstruct, the $t$ lowest-indexed active players run the instance corresponding to their subset while the remaining active players are silent. This is only efficient when $t($ or $n-t)$ is small. 


\section{References}

1. Abraham, I., Dolev, D., Gonen, R., Halpern, J.: Distributed computing meets game theory: robust mechanisms for rational secret sharing and multiparty computation. In: 25th ACM Symposium Annual on Principles of Distributed Computing, pp. 53-62. ACM Press, New York (2006)

2. Alwen, J., Katz, J., Lindell, Y., Persiano, G., Shelat, A., Visconti, I.: Collusionfree multiparty computation in the mediated model. In: Halevi, S. (ed.) CRYPTO 2009. LNCS, vol. 5677, pp. 524-540. Springer, Heidelberg (2009)

3. Alwen, J., Shelat, A., Visconti, I.: Collusion-free protocols in the mediated model. In: Wagner, D. (ed.) CRYPTO 2008. LNCS, vol. 5157, pp. 497-514. Springer, Heidelberg (2008)

4. Asharov, G., Lindell, Y.: Utility dependence in correct and fair rational secret sharing. In: Halevi, S. (ed.) CRYPTO 2009. LNCS, vol. 5677, pp. 559576. Springer, Heidelberg (2009), A full version, containing additional results: http://eprint.iacr.org/209/373

5. Blakley, G.: Safeguarding cryptographic keys. In: Proc. AFIPS National Computer Conference, vol. 48, pp. 313-317 (1979)

6. Dodis, Y.: Efficient construction of (distributed) verifiable random functions. In: Desmedt, Y.G. (ed.) PKC 2003. LNCS, vol. 2567, pp. 1-17. Springer, Heidelberg (2002)

7. Dodis, Y., Rabin, T.: Cryptography and game theory. In: Nisan, N., Roughgarden, T., Tardos, E., Vazirani, V. (eds.) Algorithmic Game Theory, pp. 181-207. Cambridge University Press, Cambridge (2007)

8. Dodis, Y., Yampolskiy, A.: A verifiable random function with short proofs and keys. In: Vaudenay, S. (ed.) PKC 2005. LNCS, vol. 3386, pp. 416-431. Springer, Heidelberg (2005)

9. Fuchsbauer, G., Katz, J., Naccache, D.: Efficient rational secret sharing in standard communication networks, http://eprint.iacr.org/2008/488

10. Gordon, S.D., Hazay, C., Katz, J., Lindell, Y.: Complete fairness in secure twoparty computation. In: 40th Annual ACM Symposium on Theory of Computing (STOC), pp. 413-422. ACM Press, New York (2008)

11. Gordon, S.D., Katz, J.: Rational secret sharing, revisited. In: De Prisco, R., Yung, M. (eds.) SCN 2006. LNCS, vol. 4116, pp. 229-241. Springer, Heidelberg (2006)

12. Gordon, S.D., Katz, J.: Partial fairness in secure two-party computation (2008), http://eprint.iacr.org/2008/206

13. Halpern, J., Teague, V.: Rational secret sharing and multiparty computation: Extended abstract. In: 36th Annual ACM Symposium on Theory of Computing (STOC), pp. 623-632. ACM Press, New York (2004)

14. Izmalkov, S., Lepinski, M., Micali, S.: Verifiably secure devices. In: Canetti, R. (ed.) TCC 2008. LNCS, vol. 4948, pp. 273-301. Springer, Heidelberg (2008)

15. Izmalkov, S., Micali, S., Lepinski, M.: Rational secure computation and ideal mechanism design. In: 46th Annual Symposium on Foundations of Computer Science (FOCS), pp. 585-595. IEEE, Los Alamitos (2005)

16. Katz, J.: Bridging game theory and cryptography: Recent results and future directions. In: Canetti, R. (ed.) TCC 2008. LNCS, vol. 4948, pp. 251-272. Springer, Heidelberg (2008)

17. Katz, J.: Ruminations on defining rational MPC. Talk given at SSoRC, Bertinoro, Italy (2008), http://www.daimi.au.dk/ jbn/SSoRC2008/program 
18. Kol, G., Naor, M.: Cryptography and game theory: Designing protocols for exchanging information. In: Canetti, R. (ed.) TCC 2008. LNCS, vol. 4948, pp. 320-339. Springer, Heidelberg (2008)

19. Kol, G., Naor, M.: Games for exchanging information. In: 40th Annual ACM Symposium on Theory of Computing (STOC), pp. 423-432. ACM Press, New York (2008)

20. Lepinski, M., Micali, S., Peikert, C., Shelat, A.: Completely fair SFE and coalitionsafe cheap talk. In: 23rd ACM Symposium Annual on Principles of Dis- tributed Computing, pp. 1-10. ACM Press, New York (2004)

21. Lepinski, M., Micali, S., Shelat, A.: Collusion-free protocols. In: 37th Annual ACM Symposium on Theory of Computing (STOC), pp. 543-552. ACM Press, New York (2005)

22. Lepinski, M., Micali, S., Shelat, A.: Fair-zero knowledge. In: Kilian, J. (ed.) TCC 2005. LNCS, vol. 3378, pp. 245-263. Springer, Heidelberg (2005)

23. Lysyanskaya, A.: Unique signatures and verifiable random functions from the DHDDH separation. In: Yung, M. (ed.) CRYPTO 2002. LNCS, vol. 2442, pp. 597-612. Springer, Heidelberg (2002)

24. Lysyanskaya, A., Triandopoulos, N.: Rationality and adversarial behavior in multi-party computation. In: Dwork, C. (ed.) CRYPTO 2006. LNCS, vol. 4117, pp. 180-197. Springer, Heidelberg (2006)

25. Micali, S., Rabin, M.O., Vadhan, S.P.: Verifiable random functions. In: 40th Annual Symposium on Foundations of Computer Science (FOCS), pp. 120-130. IEEE, Los Alamitos (1999)

26. Miclai, S., Shelat, A.: Truly rational secret sharing. In: Reingold, O. (ed.) TCC 2009. LNCS, vol. 5444, pp. 54-71. Springer, Heidelberg (2009)

27. Ong, S.J., Parkes, D., Rosen, A., Vadhan, S.: Fairness with an honest minority and a rational majority. In: Reingold, O. (ed.) TCC 2009. LNCS, vol. 5444, pp. 36-53. Springer, Heidelberg (2009)

28. Shamir, A.: How to share a secret. Communications of the ACM 22(11), 612-613 (1979)

\section{A Verifiable Random Functions (VRFs)}

A VRF is a keyed function whose output is "random-looking" but can still be verified as correct, given an associated proof. The notion was introduced by Micali, Rabin, and Vadhan [25], and various constructions in the standard model are known [25, 6, 23, 8. The definition we use is stronger than the "standard" one in that it includes a uniqueness requirement on the proof as well, but the constructions of 6,8 achieve it. (Also, we use VRFs only as a stepping stone to our construction based on trapdoor permutations; see Section [3.1.)

Definition 5. A verifiable random function (VRF) with range $\mathcal{R}=\left\{\mathcal{R}_{k}\right\}$ is a tuple of probabilistic polynomial-time algorithms (Gen, Eval, Prove, Vrfy) such that the following hold:

Correctness: For all $k$, any $(p k, s k)$ output by Gen $\left(1^{k}\right)$, the algorithm $\mathrm{Eval}_{s k}$ maps $k$-bit inputs to the set $\mathcal{R}_{k}$. Furthermore, for any $x \in\{0,1\}^{k}$ we have $\operatorname{Vrfy}_{p k}\left(x, \operatorname{Eval}_{s k}(x)\right.$, Prove $\left._{s k}(x)\right)=1$. 
Verifiability: For all $(p k, s k)$ output by $\operatorname{Gen}\left(1^{k}\right)$, there does not exist a tuple $\left(x, y, y^{\prime}, \pi, \pi^{\prime}\right)$ with $y \neq y^{\prime}$ and $\operatorname{Vrfy}_{p k}(x, y, \pi)=1=\operatorname{Vrfy}_{p k}\left(x, y^{\prime}, \pi^{\prime}\right)$.

Unique proofs: For all $(p k, s k)$ output by $\operatorname{Gen}\left(1^{k}\right)$, there does not exist a tuple $\left(x, y, \pi, \pi^{\prime}\right)$ with $\pi \neq \pi^{\prime}$ and $\operatorname{Vrfy}_{p k}(x, y, \pi)=1=\operatorname{Vrfy}_{p k}\left(x, y, \pi^{\prime}\right)$.

Pseudorandomness: Consider the following experiment involving an adversary $\mathcal{A}$ :

1. Generate $(p k, s k) \leftarrow \operatorname{Gen}\left(1^{k}\right)$ and give $p k$ to $\mathcal{A}$. Then $\mathcal{A}$ adaptively queries a sequence of strings $x_{1}, \ldots, x_{\ell} \in\{0,1\}^{k}$ and is given $y_{i}=$ $\operatorname{Eval}_{s k}\left(x_{i}\right)$ and $\pi_{i}=\operatorname{Prove}_{s k}\left(x_{i}\right)$ in response to each such query $x_{i}$.

2. $\mathcal{A}$ outputs a string $x \in\{0,1\}^{k}$ subject to the restriction $x \notin\left\{x_{1}, \ldots, x_{\ell}\right\}$.

3. $A$ random bit $b \leftarrow\{0,1\}$ is chosen. If $b=0$ then $\mathcal{A}$ is given $y=\mathrm{Eval}_{s k}(x)$; if $b=1$ then $\mathcal{A}$ is given a random $y \leftarrow \mathcal{R}_{k}$.

4. $\mathcal{A}$ makes more queries as in step 2, as long as none of these queries is equal to $x$.

5. At the end of the experiment, $\mathcal{A}$ outputs a bit $b^{\prime}$ and succeeds if $b^{\prime}=b$.

We require that the success probability of any $\mathrm{PPT}$ adversary $\mathcal{A}$ is $\frac{1}{2}+\operatorname{negl}(k)$. 